Cite this: Phys. Chem. Chem. Phys., 2013 15, 19065

Received 16th July 2013,

Accepted 20th September 2013

DOI: $10.1039 / \mathrm{c} 3 \mathrm{cp} 52970 \mathrm{c}$

www.rsc.org/pccp

\title{
Mesogen polarity effects on biaxial nematics. Centrally located dipoles $\uparrow$
}

\author{
Lara Querciagrossa, Matteo Ricci, Roberto Berardi* and Claudio Zannoni
}

\begin{abstract}
We investigate the phase organisation of thermotropic biaxial Gay-Berne (GB) mesogens yielding a biaxial nematic $\left(\mathrm{N}_{\mathrm{b}}\right)$ phase upon endowing them with a central point dipole. We study the effects of changing the strength and orientation of the dipole on the phase behaviour, and in particular we examine, using molecular dynamics (MD) simulations, the possibility of improving the stability of the $\mathrm{N}_{\mathrm{b}}$ phase. After mapping the boundaries of the $\mathrm{N}_{\mathrm{b}}$ phase, we find that the strength of the embedded dipole is the parameter with the strongest influence on the mesogenic properties, while its orientation plays a minor role. For these central dipole systems, we find that the $\mathrm{N}_{\mathrm{b}}$ phase organisation is stable only for mesogens with relatively weak dipole moments, while it disappears if electrostatic interactions become comparable in magnitude with dispersion interactions.
\end{abstract}

\section{Introduction}

Biaxial nematics $\left(\mathrm{N}_{\mathrm{b}}\right)$ are currently of great interest in applied liquid crystals research (LC) ${ }^{1-5}$ since these anisotropic fluids have, differently from the usual uniaxial variety, the characteristic of having two, rather than just one, directions of preferential alignment (directors) that can be independently controlled by external fields and surface treatments. A feature of particular interest for LC display technology is the significantly faster switching time of the secondary director that is expected for these materials ${ }^{6}$ and that could be exploited, provided suitable thermotropic $\mathrm{N}_{\mathrm{b}}$ materials become available. Biaxial nematics should in principle form as easily as uniaxial ones and indeed their existence was predicted 40 years ago by mean field theory ${ }^{7}$ and confirmed by lattice ${ }^{8}$ and off-lattice ${ }^{9,10}$ computer simulations. ${ }^{4}$ However, reality has revealed them to be quite different: ${ }^{2,3,11}$ while $\mathrm{N}_{\mathrm{b}}$ have been found at an early stage in lyotropic, ${ }^{1}$ polymeric $\mathrm{LC}^{12-14}$ and, recently, in elastomeric $\mathrm{LC}^{15,16}$ and colloidal $^{17}$ systems, low molar mass thermotropic materials have defied synthetic chemistry until bent-core, ${ }^{18,19}$ tetrapodes $^{20}$ and similar complex mesogens have quite convincingly shown $\mathrm{N}_{\mathrm{b}}$ phases.

Unfortunately, current $\mathrm{N}_{\mathrm{b}}$ materials are unsuitable for practical display applications as they have, e.g. too high transition temperatures or inappropriate viscosities. It should of course be possible, at

Department of Industrial Chemistry "Toso Montanari", University of Bologna, vialie Risogimento 4, I-40136 Bologna, Italy.E-mail: roberto.berardic@unibo.it; Fax: +39-051-20-93690; Tel: +39-051-20-93698

$\dagger$ Electronic supplementary information (ESI) available. See DOI: 10.1039/ c3cp52970c least in principle, to modify current $\mathrm{N}_{\mathrm{b}}$ mesogens and design better ones, but the few experimental findings are not yet supported by systematic structure-phase behaviour guidelines that, in the absence of a complete theory, probably impossible to realise, would be very important to avoid a brute force trial and error approach.

Computer simulations can be particularly useful in this respect ${ }^{4}$ as they allow a specific investigation of the effects that selected molecular features might have on the phase behaviour. Here, we study, with this aim in mind, the effects of a molecular dipole positioned at the centre of the mesogen. The electric dipole is a particular interesting element in chemical design ${ }^{21}$ and it has been studied theoretically by various authors for uniaxial elongated, ${ }^{22-28}$ and $\operatorname{discotic}^{29,30}$ moieties since, by suitable functional substitution, it can experimentally be placed at selected positions and orientations in an existing mesogen, controlling also, to some extent, its strength from the few Debye of $n$-alkane cyanobiphenyls $(n \mathrm{CB})^{31}$ to very large values, even above $20 \mathrm{D}$ of carborane LC. ${ }^{32,33}$ One important effect that the dipole has is that of contributing to the dielectric susceptivity of a material and its anisotropy, thus determining the alignment of a LC parallel or perpendicular to an applied electric field, ${ }^{34}$ a key property for displays. On the other hand, dipoles are also quite challenging and difficult to deal with in terms of predicting their collective behaviour, ${ }^{22}$ making common sense rather useless. For instance, we have shown in the past that simply shifting a longitudinal molecular dipole from the central to a near-terminal position in a uniaxial mesogen can dramatically change the resulting smectic phase from a $\mathrm{S}_{\mathrm{m}} \mathrm{A}$ with random distribution of dipole orientations to a $\mathrm{S}_{\mathrm{m}} \tilde{\mathrm{A}}$ organisation with alternating stripes of dipoles with coherent orientation. ${ }^{22}$ More generally it is not easy to predict the effect 
that the dipole has on the relative stability of the uniaxial nematic, biaxial nematic or smectic phases and the resulting effects on the narrowing or widening of the range of existence of the target biaxial nematic.

The paper is organised as follows: in the next two sections the biaxial dipolar Gay-Berne model is presented and technical details of the molecular dynamics simulations performed are described. Then in the following section, the simulation results are reported and discussed, while in the final section we draw some conclusions on the use of dipoles to tune phase biaxiality in nematic fluids.

\section{Model and technical details}

We have modelled the mesogenic molecules at the coarsegrained level as rigid ellipsoidal particles characterised by positional and orientational degrees of freedom, free to move in space and interacting with a pair potential that is the sum of two terms, a Gay-Berne (GB) energy $U_{\mathrm{GB}}$ and an electrostatic $U_{\mathrm{el}}$ contribution:

$$
U_{12}=U_{\mathrm{GB}}+U_{\mathrm{el}}
$$

The first pair interaction term is the attractive-repulsive energy for two rigid biaxial GB ellipsoids: $:^{4,35-37}$

$U_{\mathrm{GB}}\left(\mathbf{r}, \omega_{1}, \omega_{2}\right)=4 \varepsilon_{0} \varepsilon\left(\mathbf{r}, \omega_{1}, \omega_{2}\right)\left[u^{12}\left(\mathbf{r}, \omega_{1}, \omega_{2}\right)-u^{6}\left(\mathbf{r}, \omega_{1}, \omega_{2}\right)\right]$.

Here, $\mathbf{r} \equiv \mathbf{r}_{2}-\mathbf{r}_{1}$ is the intermolecular vector connecting the centres of mass of the two GB particles, whose modulus is the intermolecular distance $r$, while the orientations of the two interacting ellipsoids $\omega_{i}$ are given here by a four components quaternion $\left(Q_{i}\right),{ }^{4,6}$ equivalent to the classical representation in terms of three Euler angles, ${ }^{38}$ but much more convenient from the computational point of view. ${ }^{6,39}$

The function $u\left(\mathbf{r}, \omega_{1}, \omega_{2}\right)=\sigma_{c} /\left(\mathbf{r}-\sigma\left(\mathbf{r}, \omega_{1}, \omega_{2}\right)+\sigma_{c}\right)$ contains the anisotropic contact term $\sigma\left(\mathbf{r}, \omega_{1}, \omega_{2}\right)$ which approximates the geometrical "contact distance" between two ellipsoids and depends on the axes lengths $\sigma_{x}, \sigma_{y}$ and $\sigma_{z}$. The interaction term $\varepsilon\left(\mathbf{r}, \omega_{1}, \omega_{2}\right)$ defines the potential well depth depending on the orientations and on the interaction parameters $\varepsilon_{x}, \varepsilon_{y}$ and $\varepsilon_{z}$, which are directly related to the well depths for two GB particles approaching with fixed parallel orientations along the three Cartesian directions.

Embedding two equal electric charges $\pm q$, of same magnitude and opposite sign, symmetrically positioned at $\pm \mathbf{r}_{q}$ with respect to the particle centre (such that the distance $d=2\left\|\mathbf{r}_{q}\right\|$ is much smaller than the Gay-Berne particle dimensions $\sigma_{i}$ ) allows us to effectively model a central point electric dipole $\mu=q d$ at an arbitrarily chosen orientation. Here, we used $d=$ $0.2 \sigma_{0}$ (see Table 1 ).

The total electrostatic interaction $U_{\mathrm{el}}$ between two GB particles 1,2 becomes:

$$
U_{\mathrm{el}}=\sum_{a \in 1, b \in 2} \frac{q_{a} q_{b}}{r_{a b}}
$$

where the sum runs over the charges $q_{a}$ of particle 1 and $q_{b}$ of particle 2 (at distance $r_{a b}$ ), and we use a CGS notation for the electrostatic energy (see ref. 40 for further details). To compute electrostatic interactions, we have employed the reaction-field like method reported by Tironi et al. ${ }^{40}$ The suitability of this approach for our samples has been checked by comparing the MD results of selected state points with those obtained from a full Ewald calculation, ${ }^{22}$ more rigorous but much more demanding in terms of computer resources. We found the two methods to give comparable results (possibly because we did not observe any long-range polar organisation).

Using a previously established notation, ${ }^{4,10}$ the molecular units of mass, length and energy are indicated as $m_{0}, \sigma_{0}$ and $\varepsilon_{0}$ (not to be confused with the vacuum permittivity $\varepsilon_{0}$ ). All quantities have been employed in the dimensionless form: e.g. temperature $T^{*} \equiv T / T_{0}=T /\left(k_{\mathrm{B}}{ }^{-1} \varepsilon_{0}\right)$, pressure $P^{*} \equiv P / P_{0}=$ $P /\left(\varepsilon_{0}{ }^{-1} \sigma_{0}{ }^{3}\right)$, time $t^{*} \equiv t / t_{0}=t /\left(m_{0} \sigma_{0}{ }^{2} \varepsilon_{0}{ }^{-1}\right)^{1 / 2}$, electrostatic charge $q^{*} \equiv q / q_{0}=q /\left(\varepsilon_{0} \sigma_{0}\right)^{1 / 2}$ and dipole moment $\mu^{*} \equiv \mu / \mu_{0}=\mu /\left(\varepsilon_{0} \sigma_{0}^{3}\right)^{1 / 2}$ (with $\mu_{0}$ not to be confused with the vacuum permeability). Using these definitions and taking as units values for length

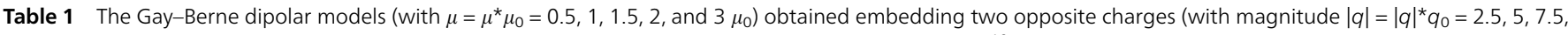

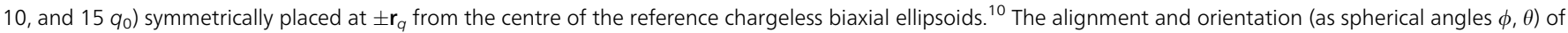

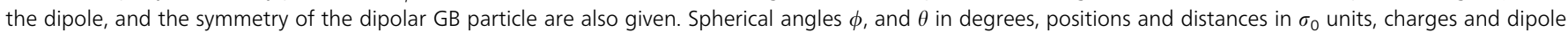
moments in $q_{0}=\left(\varepsilon_{0} \sigma_{0}\right)^{1 / 2}$ and $\mu_{0}=\left(\varepsilon_{0} \sigma_{0}{ }^{3}\right)^{1 / 2}$ units, respectively

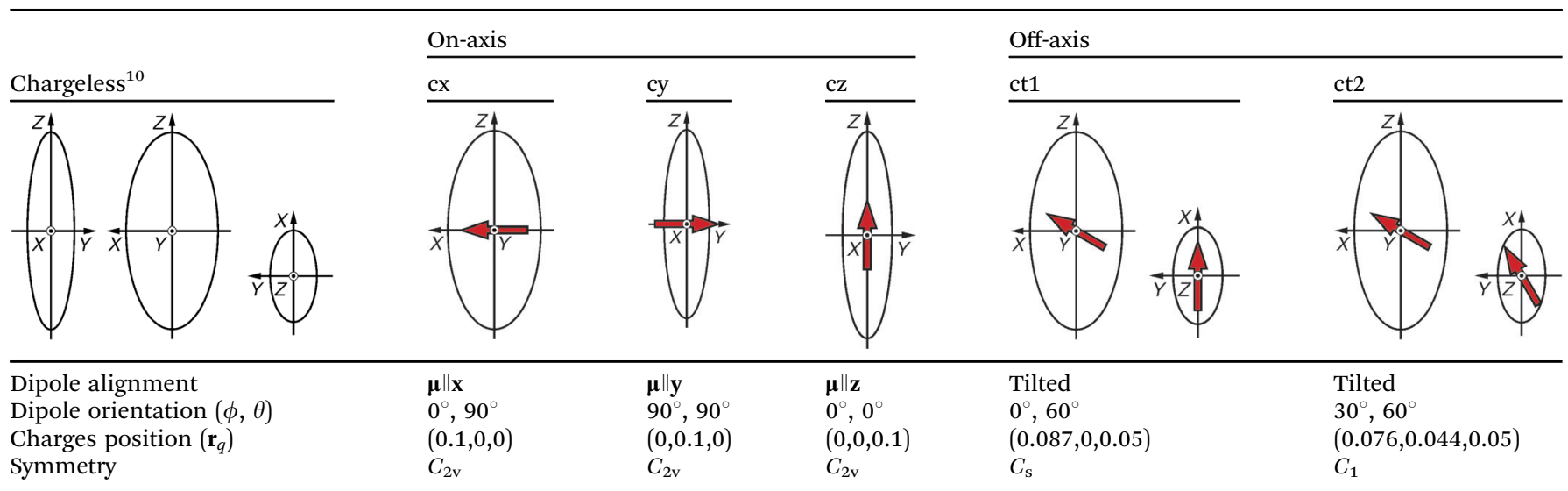


and energy $\sigma_{0}=5 \times 10^{-10} \mathrm{~m}, \varepsilon_{0}=1.4 \times 10^{-21} \mathrm{~J}$, and $m_{0}=4.8 \times$ $10^{-22} \mathrm{~kg}$, typical of a low molar mass mesogen such as $8 \mathrm{CB}$, we have that $T^{*}=1$ roughly corresponds to $T=100 \mathrm{~K}, t^{*}=1$ to $t_{0}=3 \times 10^{-10} \mathrm{~s}, q^{*}=1$ to $q_{0}=8.767 \times 10^{-21} \mathrm{C}$ and $\mu^{*}=1$ to $\mu_{0}=1.314 D=4.383 \times 10^{-30} \mathrm{C} \mathrm{m}$.

Since we are interested in studying the effects that an electric point dipole has on a $\mathrm{N}_{\mathrm{b}}$ phase, and obtaining such a phase is in itself a non-trivial problem, we have chosen as reference dipole-less model the mesogenic GB particles studied in ref. 10 that, as we have already shown, give a stable biaxial nematic phase. More specifically, we have chosen each biaxial ellipsoidal GB particle to have the following parameterisation: mass $m=1 m_{0}$, axes $\sigma_{x}=1.4 \sigma_{0}, \sigma_{y}=0.714 \sigma_{0}, \sigma_{z}=3 \sigma_{0}, \sigma_{c}=0.714$ $\sigma_{0}$ and interaction parameters $\varepsilon_{x}=1.7 \varepsilon_{0}, \varepsilon_{y}=1 \varepsilon_{0}$ and $\varepsilon_{z}=0.2 \varepsilon_{0}$. Table 1 summarises dipole moments, orientations and overall symmetry of the biaxial GB particles studied. As cutoff radii for the GB and electrostatic potentials we have used $r_{\mathrm{GB}}=4 \sigma_{0}$, and $r_{\mathrm{el}}=6 \sigma_{0}$, respectively. The relative permittivity of the surrounding medium was 3 .

Equilibrium molecular organisations for each dipolar model system have been obtained at a number of temperatures using molecular dynamics (MD) simulations run with an in house written quaternion based code, ${ }^{6,41,42}$ at specific dimensionless pressure and temperature maintained constant by means of standard Berendsen barostat and thermostat. ${ }^{43}$ The production run lengths were typically around 300000 time steps (and never shorter than 200000 time steps) using a time step $\Delta t^{*}=0.001$ which, referring to the above units, corresponds to $\approx 0.3 \mathrm{ps}$, and in turn to equilibrium trajectories of the order of $90 \mathrm{~ns}$ each. Considering the various models and dipole moments the total number of MD simulations we have performed is well above 250 .

We have considered an orthorhombic sample box, endowed with full periodic boundary conditions, whose sides were allowed to change independently, so as to accommodate more easily the smectic structures formed at the lowest temperatures. The systems considered here were composed of $N=1024$ identical biaxial GB particles, a size large enough to explore the general phase behaviour for the several dipolar models we wish to consider, while being still manageable in terms of computer resources.

Each system has been studied at a constant dimensionless pressure $P^{*}=8$ and for temperatures ranging from $T^{*}=2.6$ to $T^{*}=3.6$, starting with the lowest dipole strength case. Each subsequent case with progressively increasing dipole moment has then been studied as a new cooling down sequence started from the equilibrated isotropic phase of the previous system. Here we report and discuss the main results of this extensive investigation, while full details are given in a set of tables in the ESI. $†$

\section{Phase and structure assignment}

The protocol we have used for assigning the resulting phase of each MD sample is based on a combined analysis of orientational order parameters, positional correlation functions and on a visual inspection of the final equilibrated configuration at each temperature. ${ }^{4,44}$

The average second rank orientational order parameters, which are the first terms of the expansion of the single-particle orientational distribution in an orthogonal basis set of symmetrised Wigner rotation matrices $R_{m, n}^{2}$, have been computed from the eigenvalues of cartesian ordering matrices using the now standard algorithm introduced in ref. 8 and 10. In particular, the Maier-Saupe order parameter is:

$$
\left\langle R_{0,0}^{2}\right\rangle=\left\langle\frac{3}{2}(\mathbf{z} \cdot \mathbf{n})^{2}-\frac{1}{2}\right\rangle \text {. }
$$

The order parameter $\left\langle R_{0,0}^{2}\right\rangle \equiv\left\langle P_{2}\right\rangle$, ranges between zero (isotropic) and one (completely aligned), and it is typically used for characterising uniaxial phases. For biaxial liquid crystals the most informative orientational order parameter is ${ }^{8,9}$

$$
\left\langle R_{2,2}^{2}\right\rangle=\left\langle\frac{1}{4}\left[(\mathbf{x} \cdot \mathbf{l})^{2}-(\mathbf{x} \cdot \mathbf{m})^{2}+(\mathbf{y} \cdot \mathbf{l})^{2}-(\mathbf{y} \cdot \mathbf{m})^{2}\right]\right\rangle,
$$

where $\mathbf{x}, \mathbf{y}$ and $\mathbf{z}$ are the three molecular frame axes and $\mathbf{l}, \mathbf{m}$ and $\mathbf{n}$ are the overall mesophase directors, with $\mathbf{n}$ being the principal director, and $\mathbf{m}$ the secondary biaxial one (perpendicular to $\mathbf{n}$ and $\mathbf{l}=\mathbf{m} \times \mathbf{n})$, while the angular brackets $\langle\cdots\rangle$ indicate ensemble averages. The order parameter $\left\langle R_{2,2}^{2}\right\rangle$ unambiguously identifies biaxial phases formed of biaxial molecules, ${ }^{8}$ where $\mathbf{x}$ and $\mathbf{y}$ axes are also macroscopically aligned, whenever it takes values larger than zero (uniaxial limit) up to one half (completely biaxial limit). In the framework of our MD simulations, with samples of relatively small size and full periodic boundaries, we have always observed uniformly ordered monodomains along arbitrary directions, rather than polydomains. This is to some extent advantageous, but the finite size of the samples (formed of $N$ particles) also reduces our ability of observing first order transitions while the statistical uncertainty, proportional to $1 / \sqrt{N}$, also provides a bound for the lowest values of the order parameters attainable in the isotropic phase.

Taking into account these effects, during our cooling down MD runs, starting from an isotropic sample, we have arbitrarily identified the formation of a nematic fluid whenever $\left\langle R_{0,0}^{2}\right\rangle \geq$ 0.3 and, similarly, the spontaneous onset of biaxial phases with ordering along a direction $\mathbf{m}$ perpendicular to $\mathbf{n}$, for $\left\langle R_{2,2}^{2}\right\rangle \geq$ 0.1. It should be noted that even though the observation of orientational order parameters being larger than specific thresholds is a necessary condition to assign uniaxial and biaxial phases, not only nematic, but also smectic or crystals phases may match these criteria, so further tests are necessary to identify positional ordering if present.

We have verified first that all nematic phases are not solid or glassy by monitoring, as in other cases, ${ }^{45,46}$ the time dependence of the molecular mean square displacements along the different directions $\alpha$ of the director frame, $\left(\Delta r_{\alpha}\right)^{2}(t)$, and checking that particles diffuse away from an arbitrarily chosen initial origin with a linear behaviour of the mean square displacements, so that the diffusion coefficient, proportional to the slope of the curve, is comparable to that of nematics 
formed by similar GB particles. Even if a detailed summary of these dynamics indicators for the various nematic phases of our dipolar GB model goes beyond the scope of this work, which was focused on structure, we have observed that in all cases the nematic organisations are actually fluid since they have diffusion coefficients which are roughly only $20-30 \%$ lower than those measured for the corresponding isotropic liquid. On the other hand, for the layered, low-temperature, organisations these values decrease by more than one order of magnitude. ${ }^{47}$

To discriminate the nematic phases from the layered structures typical of smectics (or solids) we have examined positional pair correlation functions. The first one we have computed is the standard radial pair correlation $g_{0}(r)$, giving the average probability of finding the centre of mass of any two molecules separated by a distance $r$, using as reference the value expected from an uniform distribution

$$
g_{0}(r)=\frac{1}{4 \pi r^{2} \rho}\left\langle\delta\left(r-r_{12}\right)\right\rangle_{12},
$$

where $\rho$ is the mean number density of the sample and $\langle\cdots\rangle_{12}$ denotes an average with respect to the pair distribution function. In MD simulations this function is calculated as a discrete histogram, and smectic phases can be identified if a characteristic sequence of well defined maxima and minima in $g_{0}(r)$ reveals the presence of strong positional correlations extending over the first shell of neighbouring molecules.

As a next step and to probe the formation and size of polar clusters, i.e. of regions with essentially parallel dipoles, ${ }^{48,49}$ we have computed axis-axis orientational pair correlations defined as:

$$
C_{a b}(r)=\frac{1}{4 \pi r^{2} \rho}\left\langle\delta\left(r-r_{12}\right)(\mathbf{a} \cdot \mathbf{b})\right\rangle_{12}
$$

where $\mathbf{a}, \mathbf{b}=\mathbf{x}, \mathbf{y}, \mathbf{z}$ are the unit vectors defining the orientations of the molecular axes. These functions provide information on the probability of observing a pair of molecules at distance $r$ with a certain mutual orientation of a pair of axes, and can be used to calculate the dipole-dipole correlations as follows:

$$
\begin{aligned}
C_{\mu \mu}(r)= & \frac{1}{4 \pi r^{2} \rho}\left\langle\delta\left(r-r_{12}\right)\left(\mu_{1} \cdot \mu_{2}\right)\right\rangle_{12} \\
= & \mu_{x}^{2} C_{x x}(r)+\mu_{y}^{2} C_{y y}(r)+\mu_{z}^{2} C_{z z}(r) \\
& +2 \mu_{x} \mu_{y} C_{x y}(r)+2 \mu_{x} \mu_{z} C_{x z}(r)+2 \mu_{y} \mu_{z} C_{y z}(r) .
\end{aligned}
$$

The phase assignment to nematic or smectic has also of course been supported by a direct visual inspection of equilibrium molecular configurations, where each elongated GB particle is colour coded according to its orientation using a mapping attempting to highlight the existence of polar (and biaxial) domains. We notice that the GB particles in some of the lowest temperature samples showed a certain degree of orientational tilt with respect to the layers normal (typically $\approx 5$ to $10^{\circ}$, see also ref. 24); however, since here we focus on the already complex task of discussing biaxial nematic phases and their range of existence, a specific study of these issues has not been systematically pursued.

\section{Results and discussion}

We now discuss the specific details of our MD results and rather than examining the various dipole configurations one by one; we show together the results for the dipole aligned along one of the three axes of the ellipsoidal particle and then those with a tilted orientation. We use for the two groups of models the notation provided in Table 1.

\subsection{Central dipole along a molecular axis}

The first group of three models is that of GB ellipsoids with a central dipole aligned along one of the molecular axes (i.e. see the models cx, cy and cz of Table 1).

Summarising the results, these systems show a sequence of phases from isotropic (I) to nematics $\left(\mathrm{N}, \mathrm{N}_{\mathrm{b}}\right)$ to smectics $\left(\mathrm{S}_{\mathrm{b}}\right.$, see Table 2) with no direct change from isotropic to $\mathrm{N}_{\mathrm{b}}$, or to $\mathrm{S}_{\mathrm{b}}$ (for the range of dipoles studied), in this sense similar to what was previously observed for uniaxial dipolar mesogens based on GB models. ${ }^{24}$

In Fig. 1 we show the overall $T^{*}, \mu^{*}$ phase diagrams obtained for these three cases (plates A, C, and E) and the ratios $\left\langle U_{\mathrm{el}}\right\rangle /\langle U\rangle$ giving the contribution of the electrostatic energy with respect to the total potential $\langle U\rangle=\left\langle U_{\mathrm{GB}}\right\rangle+\left\langle U_{\mathrm{el}}\right\rangle$ (plates B, D, and F). The ratios $\left\langle U_{\mathrm{el}}\right\rangle /\langle U\rangle$ for the central models with $\mu^{*}=0.5$ are practically zero and overlapped with those for the chargeless system (Fig. 1B, D, and F), while for larger dipole moments the electrostatic energy contribution becomes significant. The first significant observation on the effect of introducing the dipole is that the I-N phase transition temperature is essentially constant (cy model) or only rather marginally affected by the larger electrostatic interactions (models cx, and cz), as the dipole moment $\mu^{*}$ increases. Moreover, in all cases the $\mathrm{N}_{\mathrm{b}}$, if it exists, occurs at lower temperatures than the uniaxial one.

Differently from the nematic-isotropic, the transition from

\begin{tabular}{|c|c|c|c|c|c|c|c|c|c|c|c|c|c|c|}
\hline \multirow[b]{3}{*}{$T^{*}$} & \multicolumn{14}{|l|}{$\mu^{*}$} \\
\hline & \multirow[b]{2}{*}{0} & \multicolumn{5}{|l|}{$\mathrm{cx}$} & \multicolumn{3}{|l|}{ cy } & \multicolumn{5}{|l|}{$\mathrm{cz}$} \\
\hline & & 0.5 & 1 & 1.5 & 2 & 3 & 0.5 & 1 & 1.5 & 0.5 & 1 & 1.5 & 2 & 3 \\
\hline 2.6 & $S_{\mathrm{b}}$ & $\mathrm{S}_{\mathrm{b}}$ & $\mathrm{S}_{\mathrm{b}}$ & $\mathrm{S}_{\mathrm{b}}$ & $\mathrm{S}_{\mathrm{b}}$ & $S_{b}$ & $S_{b}$ & $S_{b}$ & $\mathrm{~S}_{\mathrm{b}}$ & $\mathrm{S}_{\mathrm{b}}$ & $\mathrm{S}_{\mathrm{b}}$ & $\mathrm{S}_{\mathrm{b}}$ & $\mathrm{S}_{\mathrm{b}}$ & $\mathrm{S}_{\mathrm{b}}$ \\
\hline 2.7 & $\mathrm{~S}_{\mathrm{b}}$ & $\mathrm{S}_{\mathrm{b}}$ & $\mathrm{S}_{\mathrm{b}}$ & $S_{b}$ & $S_{b}$ & $S_{b}$ & $\mathrm{~S}_{\mathrm{b}}$ & $\mathrm{S}_{\mathrm{b}}$ & $\mathrm{S}_{\mathrm{b}}$ & $\mathrm{S}_{\mathrm{b}}$ & $\mathrm{S}_{\mathrm{b}}$ & $\mathrm{S}_{\mathrm{b}}$ & $S_{b}$ & $S_{b}$ \\
\hline 2.8 & $\mathrm{~N}_{\mathrm{b}}$ & $\mathrm{N}_{\mathrm{b}}$ & $\mathrm{N}_{\mathrm{b}}$ & $\mathrm{S}_{\mathrm{b}}$ & $\mathrm{S}_{\mathrm{b}}$ & $S_{b}$ & $\mathrm{~N}_{\mathrm{b}}$ & $S_{b}$ & $\mathrm{~S}_{\mathrm{b}}$ & $\mathrm{N}_{\mathrm{b}}$ & $\mathrm{N}_{\mathrm{b}}$ & $\mathrm{S}_{\mathrm{b}}$ & $\mathrm{S}_{\mathrm{b}}$ & $\mathrm{S}_{\mathrm{b}}$ \\
\hline 2.9 & $\mathrm{~N}_{\mathrm{b}}$ & $\mathrm{N}_{\mathrm{b}}$ & $\mathrm{N}_{\mathrm{b}}$ & $\mathrm{N}_{\mathrm{b}}$ & $S_{b}$ & $S_{b}$ & $\mathrm{~N}_{\mathrm{b}}$ & $\mathrm{N}_{\mathrm{b}}$ & $\mathrm{S}_{\mathrm{b}}$ & $\mathrm{N}_{\mathrm{b}}$ & $\mathrm{N}_{\mathrm{b}}$ & $\mathrm{N}_{\mathrm{b}}$ & $S_{b}$ & $\mathrm{~S}_{\mathrm{b}}$ \\
\hline 3.0 & $\mathrm{~N}$ & $\mathrm{~N}$ & $\mathrm{~N}$ & $\mathrm{~N}$ & $S_{b}$ & $S_{b}$ & $\mathrm{~N}$ & $\mathrm{~N}$ & $\mathrm{~S}_{\mathrm{b}}$ & $\mathrm{N}$ & $\mathrm{N}$ & $\mathrm{N}$ & $\mathrm{N}$ & $\mathrm{S}_{\mathrm{b}}$ \\
\hline 3.1 & $\mathrm{~N}$ & $\mathrm{~N}$ & $\mathrm{~N}$ & $\mathrm{~N}$ & $\mathrm{~N}$ & $S_{b}$ & $\mathrm{~N}$ & $\mathrm{~N}$ & $\mathrm{~S}_{\mathrm{b}}$ & $\mathrm{N}$ & $\mathrm{N}$ & $\mathrm{N}$ & $\mathrm{N}$ & $\mathrm{S}_{\mathrm{b}}$ \\
\hline 3.2 & $\mathrm{~N}$ & $\mathrm{~N}$ & $\mathrm{~N}$ & $\mathrm{~N}$ & $\mathrm{~N}$ & $S_{b}$ & $\mathrm{~N}$ & $\mathrm{~N}$ & $\mathrm{~N}$ & $\mathrm{~N}$ & $\mathrm{~N}$ & $\mathrm{~N}$ & $\mathrm{~N}$ & $\mathrm{~S}_{\mathrm{b}}$ \\
\hline 3.3 & I & I & I & I & I & $\mathrm{N}$ & I & I & I & $\mathrm{N}$ & I & I & $\mathrm{N}$ & $\mathrm{N}$ \\
\hline 3.4 & I & I & I & I & I & I & I & $\mathrm{I}$ & I & I & $\mathrm{I}$ & I & I & $\mathrm{N}$ \\
\hline 3.5 & I & I & I & I & I & I & I & I & I & I & I & I & I & I \\
\hline 3.6 & I & I & I & I & I & I & I & I & I & I & I & I & I & I \\
\hline
\end{tabular}
nematic to an orthogonal smectic (or solid) progressively shifts

Table 2 The thermotropic phases for models $c x, c y$, and cz of Table 1. Labels are: isotropic $(\mathrm{I})$, uniaxial nematic $(\mathrm{N})$, biaxial nematic $\left(\mathrm{N}_{\mathrm{b}}\right)$, and orthogonal biaxial smectic or solid $\left(S_{b}\right)$. The chargeless $\mu^{*}=0$ ref. 10 results are also given 

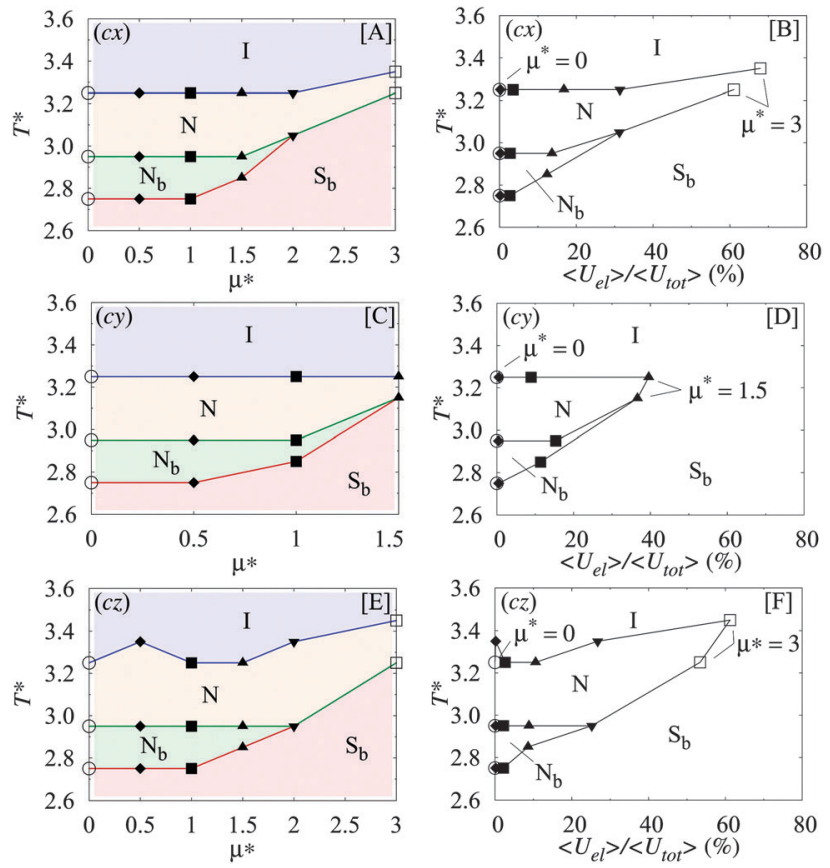

Fig. 1 The transition temperatures (see Table 2) for models $c x, c y$, and $c z$ of Table 1 against dipole moments $\mu^{*}$ (plates $\mathrm{A}, \mathrm{C}$ and $\mathrm{E}$ ), and corresponding ratios $\left\langle U_{\mathrm{el}}\right\rangle /\langle U\rangle$ (plates B, D and F). Symbols for $\mu^{\star}=0$ (circles) and $\mu^{\star}=0.5$ (filled diamonds) are typically overlapped in plates $B, D$ and $F$. Straight lines join the MD data as a guide to the eye.

to higher temperatures, considerably reducing the stability ranges of the $\mathrm{N}_{\mathrm{b}}$ and $\mathrm{N}$ fluids. Following this trend, for models cx and $\mathrm{cz}$, the $\mathrm{N}_{\mathrm{b}}$ phase disappears from the phase diagram for $1.5<\mu^{*} \leq 2$, and corresponding to this we observe a direct transition from a uniaxial nematic to a biaxial layered structure.

Upon further increasing the dipole strength, we see that for $2<\mu^{*} \leq 3$ also the uniaxial nematic is destabilised since dipole-dipole interactions strongly favour side-by-side and faceto-face configurations. In the case of the dipole pointing along the shortest molecule axis (cy model) this behaviour is even more pronounced and these changes are observed for even smaller dipole moments (see Table 2, and Fig. 1C and D).

The result, due to an increased stabilisation of smectic with respect to nematic, is quite reasonable as the central dipole can favour proper positional registration of neighbouring molecules. This stabilisation results in the onset of fairly stable orthogonal smectic structures and is probably one of the reasons for the difficulty of obtaining biaxial nematics as opposed to smectics. ${ }^{4,50}$

The group of Fig. 1B, D, and F is revealing since it shows that for models cx and cz, as the dipole-dipole interactions become greater than $\approx 10 \%$ of the total potential energy, the layered phases start eroding the nematic temperature ranges, until they eventually destroy the $\mathrm{N}_{\mathrm{b}}$ phase for $\left\langle U_{\mathrm{el}}\right\rangle /\langle U\rangle \approx 0.3$. Even the uniaxial nematic organisation practically disappears when the electrostatic interactions account for $\approx 70 \%$ of the total potential energy. A similar analysis can also be applied to the results of model cy with the difference that these threshold percentages are achieved with even smaller dipole moments
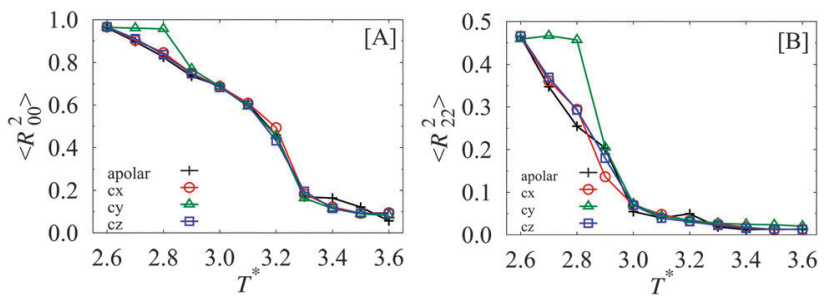

Fig. 2 The $\left\langle R_{0,0}^{2}\right\rangle$ (plate $\mathrm{A}$ ), and $\left\langle R_{2,2}^{2}\right\rangle$ (plate $\mathrm{B}$ ) order parameters for models cx (red circles), cy (green triangles), and cz (blue squares) with $\mu^{\star}=1$ (see Table 1 ). The chargeless ref. 10 results are also plotted (black ticks).
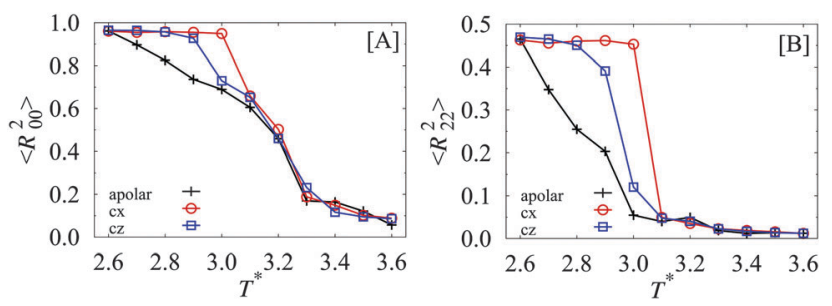

Fig. 3 The $\left\langle R_{0,0}^{2}\right\rangle$ (plate $\mathrm{A}$ ), and $\left\langle R_{2,2}^{2}\right\rangle$ (plate $\mathrm{B}$ ) order parameters for models $\mathrm{cx}$ (red circles), and cz (blue squares) with $\mu^{\star}=2$. See Fig. 2 for details.

than the cx and cz central models and nematic phases disappear for $\left\langle U_{\mathrm{el}}\right\rangle /\langle U\rangle>0.4$. This behaviour is typical of highly dipolar symmetric particles, and it is consistent with previous simulation results. $^{24}$

The plots of the order parameters of Fig. 2 and 3 show another interesting behaviour of these central dipole systems. Again, for relatively small dipole moments (e.g. $\mu^{*}=1$, Fig. 2) the temperature trends of the uniaxial $\left\langle R_{0,0}^{2}\right\rangle$ and biaxial $\left\langle R_{2,2}^{2}\right\rangle$ order parameters are practically superimposable for all dipolar models and closely follow the profile observed in the nematic phase of the chargeless reference system ${ }^{10}$ apart from deviations in the $\mathrm{S}_{\mathrm{b}}$ region for the model cy. On the other hand, for stronger dipole moments (e.g. $\mu^{*}=2$, Fig. 3) this is no longer the case, and the $\left\langle R_{0,0}^{2}\right\rangle$ order parameter profiles show well defined discontinuities across the nematic to orthogonal smectic (or solid) transitions. The phase biaxiality order parameter $\left\langle R_{2,2}^{2}\right\rangle$ plots for the cx and cz central models are affected by the orientation of the electric dipole, and the transition to a layered phase becomes much sharper.

For these nematics we have not observed any spontaneous formation of ferroelectric phases ${ }^{51}$ or even of polar cybotactic clusters, ${ }^{48}$ as found, e.g. in some bent-core mesogens where they lead to large field induced biaxiality. ${ }^{49}$ Neighbouring dipoles for our biaxial Gay-Berne particles adopt preferentially an antiferroelectric organisation, as found by analysing the histograms of the orientational axis-axis $C_{a b}(r)$ or the dipoledipole $C_{\mu \mu}(r)$ correlations. As an example, in Fig. 4A we plot the $C_{x x}(r)=C_{\mu \mu}(r)$ correlation function for model cx with $\mu^{*}=1$ in the $\mathrm{N}_{\mathrm{b}}, \mathrm{N}$, and $\mathrm{I}$ phases. We see a first minimum which corresponds to a situation where neighbouring pairs of dipoles preferentially align antiparallel, while the positive hump is for a second shell of correlated dipoles with parallel orientations. These pair correlations are stronger in the $\mathrm{N}_{\mathrm{b}}$, while they are 

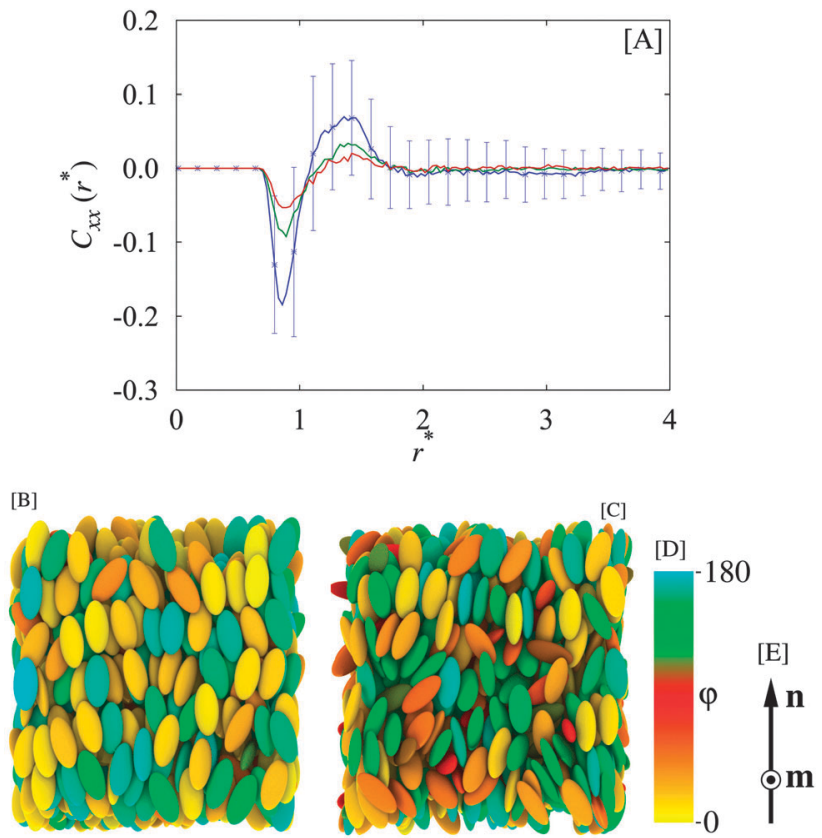

Fig. 4 The $C_{x x}(r)$ orientational correlation functions (plate A), and snapshots (plates B and C) for model $\mathrm{cx}$ with $\mu^{*}=1$ (see Table 1) at temperatures: $T^{*}=$ $2.8\left(\mathrm{~N}_{\mathrm{b}},\left\langle R_{0,0}^{2}\right\rangle=0.84,\left\langle R_{2,2}^{2}\right\rangle=0.29\right.$, blue line, and plate $\left.\mathrm{B}\right), T^{\star}=3.1\left(\mathrm{~N},\left\langle R_{0,0}^{2}\right\rangle=0.61\right.$, $\left\langle R_{2,2}^{2}\right\rangle=0.05$, green line, and plate $\left.\mathrm{C}\right)$, and $T^{\star}=3.4\left(\mathrm{I},\left\langle R_{0,0}^{2}\right\rangle=0.12,\left\langle R_{2,2}^{2}\right\rangle=0.03\right.$, red line). The error bars, plotted every 20 histogram bins, have similar amplitudes in the $N_{b}, N$, and I phases. Each GB particle has been colour coded with the palette of plate $\mathrm{D}$ according to the angle $\varphi$ between its dipole $\boldsymbol{\mu}$ and the director $\mathbf{m}$. The $\mathbf{n}$ (principal) and $\mathbf{m}$ (secondary) directors are shown in plate $\mathrm{E}$.

much weaker (and almost comparable) in the $\mathrm{N}$ and I phases where they become practically restricted to first neighbours. The statistical uncertainties in histogram populations are fairly large independent of the phase structure and degree of orientational ordering. These histograms show a feature common to all nematic systems of dipolar GB particles studied in this work, namely that these pair correlations decay to zero for distances comparable to the size of the second coordination shell, if not even smaller ones. An instance of this can also be appreciated in the snapshots of Fig. $4 \mathrm{~B}\left(\mathrm{~N}_{\mathrm{b}}\right)$ and Fig. $4 \mathrm{C}(\mathrm{N})$ where the cx GB particles have been colour coded to show the orientation of their dipole with respect to the secondary mesophase director $\mathbf{m}$. This absence of long-range orientational correlations is not surprising in view of the fact that the polar domains observed so far $^{49}$ have been assisted, differently from our ellipsoidal ones, by the convenient local stacking of the bent-core mesogens.

The influence of dipole orientation and thus the difference between the cx, cy and cz models originates from the closest distance that two ellipsoidal GB particles can attain in a configuration with side-by-side antiparallel dipoles (and consequently from how strong the electrostatic pair interaction can become with respect to the GB potential energy). This is in turn mainly governed by the length of the GB ellipsoids axes determining the distances $r_{0}=\sigma\left(r, \omega_{1}, \omega_{2}\right)$ for which $U_{\mathrm{GB}}\left(r_{0}\right)=0$. Thus, for both cx and cz models this distance $r_{0}$ is $\sigma_{y}=0.714 \sigma_{0}$ and we observe a similar mesogenic behaviour. For the cy model a side-by-side antiparallel configuration can be observed only at larger separations $\left(r_{0}=\sigma_{x}=1.4 \sigma_{0}\right)$, and the closest distance for the dipole-dipole interactions, $r_{0}=\sigma_{y}=0.714 \sigma_{0}$, is that for the end-to-end antiparallel dipoles, which gives an energy one half of the side-by-side at the same distance and orientation.

\subsection{Tilted central dipole}

We now turn to briefly discuss the case of the effect on phase behaviour of a central dipole tilted away from at least one of the particle axes. This is of course needed to check if the findings of the previous section are dependent on the assumption of dipole alignment along the molecular $\mathbf{x}, \mathbf{y}$ or $\mathbf{z}$ axes. We consider two cases (see Table 1). The first (model ct1) corresponds to a dipole tilted $60^{\circ}$ away from the long $\mathbf{z}$ axis, but remaining in the $x z$ plane. The second dipole orientation we consider (model ct2) is instead for a fully off-axis case.

The MD simulations of the two off-axis dipolar GB models ct1, and ct2, when systematically varying dipole moments $\mu^{*}$, yield the sequences of phases summarised in Table 3. In Fig. 5 we also report the phase diagrams $\left(T^{*}, \mu^{*}\right)$ and the $\left\langle U_{\mathrm{el}}\right\rangle /\langle U\rangle$ ratios that are overall similar to those obtained for the axis aligned dipole models. The corresponding profiles for the order parameters $\left\langle R_{0,0}^{2}\right\rangle$ and $\left\langle R_{2,2}^{2}\right\rangle$ for off-axis GB particles with $\mu^{*}=1$, and $\mu^{*}=2$, are plotted in Fig. 6 and 7. For models ct1, and ct2 we observe that small dipole moments $\left(\mu^{*}=1\right)$ determine order parameters trends which do not deviate substantially from the reference chargeless ones. Larger moments $\left(\mu^{*}=2\right)$ produce instead a different behaviour since the $\mathrm{N}_{\mathrm{b}}$ phase has been replaced by $\mathrm{S}_{\mathrm{b}}$.

The local organisation of neighbouring dipoles in the nematic phase is typically antiferroelectric and the $C_{a b}(r)$ histograms (not reported for conciseness) show that the dipoledipole orientational correlations average to zero for distances comparable to the particle dimensions.

Regarding particle symmetry, we notice that, even though the ct2 model does not have any symmetry plane, no evidence of chiral organisations was found in our MD samples (containing only one enantiomeric form) possibly due to the relatively small number of particles, the periodic boundaries, and fluctuations

Table 3 The thermotropic phases for models ct1 and ct2 of Table 1. See Table 2 for details

\begin{tabular}{|c|c|c|c|c|c|c|c|c|c|c|c|}
\hline \multirow[b]{3}{*}{$T^{*}$} & \multicolumn{11}{|l|}{$\mu^{*}$} \\
\hline & \multirow[b]{2}{*}{0} & \multicolumn{5}{|l|}{ ct1 } & \multicolumn{5}{|l|}{ ct2 } \\
\hline & & 0.5 & 1 & 1.5 & 2 & 3 & 0.5 & 1 & 1.5 & 2 & 3 \\
\hline 2.6 & $S_{b}$ & $S_{b}$ & $\mathrm{~S}_{\mathrm{b}}$ & $\mathrm{S}_{\mathrm{b}}$ & $S_{b}$ & $S_{b}$ & $S_{b}$ & $\mathrm{~S}_{\mathrm{b}}$ & $\mathrm{S}_{\mathrm{b}}$ & $S_{b}$ & $S_{b}$ \\
\hline 2.7 & $\mathrm{~S}_{\mathrm{b}}$ & $\mathrm{S}_{\mathrm{b}}$ & $\mathrm{S}_{\mathrm{b}}$ & $\mathrm{S}_{\mathrm{b}}$ & $\mathrm{S}_{\mathrm{b}}$ & $S_{b}$ & $\mathrm{~S}_{\mathrm{b}}$ & $\mathrm{S}_{\mathrm{b}}$ & $\mathrm{S}_{\mathrm{b}}$ & $\mathrm{S}_{\mathrm{b}}$ & $\mathrm{S}_{\mathrm{b}}$ \\
\hline 2.8 & $\mathrm{~N}_{\mathrm{b}}$ & $\mathrm{N}_{\mathrm{b}}$ & $\mathrm{N}_{\mathrm{b}}$ & $S_{b}$ & $S_{b}$ & $S_{b}$ & $\mathrm{~N}_{\mathrm{b}}$ & $\mathrm{N}_{\mathrm{b}}$ & $\mathrm{S}_{\mathrm{b}}$ & $S_{b}$ & $S_{b}$ \\
\hline 2.9 & $\mathrm{~N}_{\mathrm{b}}$ & $\mathrm{N}_{\mathrm{b}}$ & $\mathrm{N}_{\mathrm{b}}$ & $\mathrm{N}_{\mathrm{b}}$ & $\mathrm{N}$ & $\mathrm{S}_{\mathrm{b}}$ & $\mathrm{N}_{\mathrm{b}}$ & $\mathrm{N}_{\mathrm{b}}$ & $\mathrm{N}_{\mathrm{b}}$ & $\mathrm{S}_{\mathrm{b}}$ & $S_{b}$ \\
\hline 3.0 & $\mathrm{~N}$ & $\mathrm{~N}$ & $\mathrm{~N}$ & $\mathrm{~N}$ & $\mathrm{~N}$ & $\mathrm{~S}_{\mathrm{b}}$ & $\mathrm{N}$ & $\mathrm{N}$ & $\mathrm{N}$ & $\mathrm{N}$ & $\mathrm{S}_{\mathrm{b}}$ \\
\hline 3.1 & $\mathrm{~N}$ & $\mathrm{~N}$ & $\mathrm{~N}$ & $\mathrm{~N}$ & $\mathrm{~N}$ & $S_{b}$ & $\mathrm{~N}$ & $\mathrm{~N}$ & $\mathrm{~N}$ & $\mathrm{~N}$ & $S_{b}$ \\
\hline 3.2 & $\mathrm{~N}$ & $\mathrm{~N}$ & $\mathrm{~N}$ & $\mathrm{~N}$ & $\mathrm{~N}$ & I & $\mathrm{N}$ & $\mathrm{N}$ & $\mathrm{N}$ & $\mathrm{N}$ & I \\
\hline 3.3 & I & I & $\mathrm{N}$ & I & I & I & I & I & I & I & I \\
\hline 3.4 & I & I & I & I & I & I & I & I & I & I & I \\
\hline 3.5 & I & I & I & I & I & I & I & I & I & I & I \\
\hline 3.6 & I & I & I & I & I & I & I & I & I & I & I \\
\hline
\end{tabular}



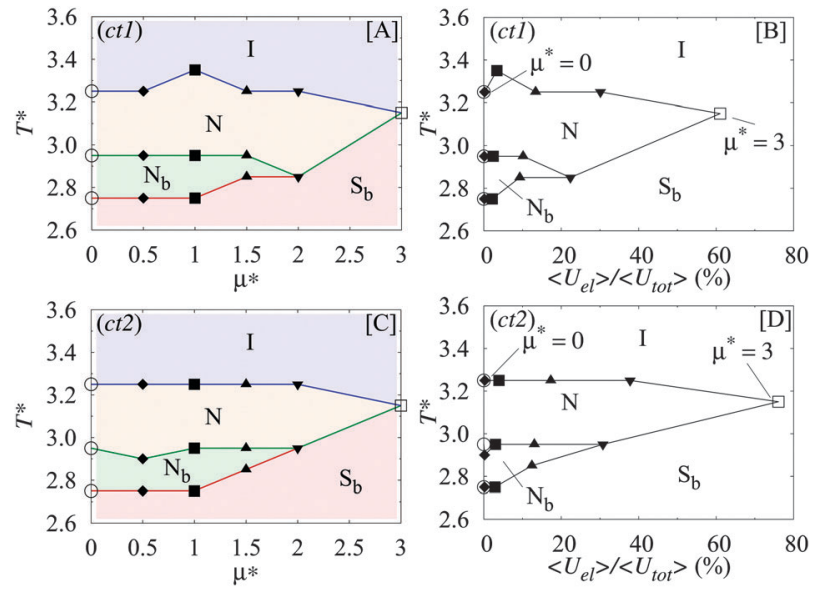

Fig. 5 The transition temperatures (see Table 3) for models ct1, and ct2 of Table 1 against dipole moments $\mu^{\star}$ (plates $\mathrm{A}$ and $\mathrm{C}$ ), and the corresponding ratios $\left\langle U_{\mathrm{el}}\right\rangle /\langle U\rangle$ (plates B and D). For further details, see the caption of Fig. 1.
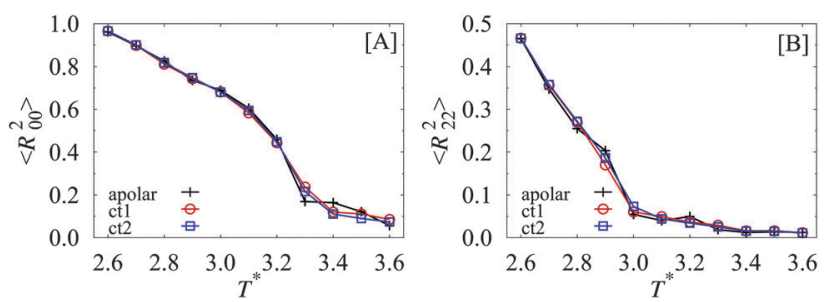

Fig. 6 The $\left\langle R_{0,0}^{2}\right\rangle$ (plate $\mathrm{A}$ ), and $\left\langle R_{2,2}^{2}\right\rangle$ (plate $\mathrm{B}$ ) order parameters for models ct1 (red circles), and ct2 (blue squares) with $\mu^{\star}=1$ (see Table 1). The chargeless ref. 10 results are also plotted (black ticks).
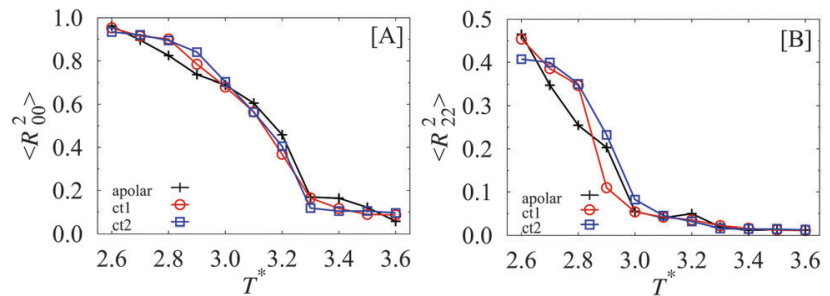

Fig. 7 The $\left\langle R_{0,0}^{2}\right\rangle$ (plate $\mathrm{A}$ ), and $\left\langle R_{2,2}^{2}\right\rangle$ (plate $\mathrm{B}$ ) order parameters for models ct1 (red circles), and ct2 (blue squares) with $\mu^{\star}=2$. See Fig. 6 for details.

large enough to overcome the small energy barriers related to the formation of chiral structures.

\section{Conclusions}

In summary, the detailed effect of adding a central dipole to a biaxial mesogen forming a biaxial nematic is quite complex, as it comes from a competition between the $\mathrm{N}, \mathrm{N}_{\mathrm{b}}$ and smectic phases, and in particular due to the changes in stability induced by the electrostatic dipolar contributions. However, we can identify a number of general features in the phase behaviour. The first is that the dipole strength appears to be the molecular parameter with the strongest influence on the phase diagram while, in comparison, the orientation of the dipole plays, at least for these central models, a minor role.
The stability of the biaxial $\mathrm{N}_{\mathrm{b}}$ phase with respect to the uniaxial one appears to be weakly affected by dipole-dipole interactions, whenever electrostatic interactions are not dominant (usually $<10 \%$ ) within the total potential energy breakdown. For intermediate $\left\langle U_{\mathrm{el}}\right\rangle /\langle U\rangle$ ratios $(\approx 30 \%)$ the $\mathrm{N}_{\mathrm{b}}$ phase becomes unstable. When electrostatic interactions become very strong (typically with a contribution to the total potential energy larger than $60-80 \%$ ), any kind of nematic organisation, both uniaxial and biaxial, tends to disappear. We notice that while we found uniaxial nematics, in no case the smectic phases formed by biaxial dipolar GB mesogens are uniaxial. Moreover, we did not find any evidence of long range polar domains, and the typical dipole-dipole short range organisation was an antiferroelectric one.

As a side remark we notice that the dipolar GB particles behaved in all cases as calamitic rod-like mesogens as the molecular axis presenting the highest degree of orientational ordering was in all cases the $z$ one (corresponding to the largest $\sigma_{i}$ length). In addition, we did not observe columnar organisations.

As for the initial question of molecular design, i.e. if it is conceivable to tune the strength of the central dipole to increase the stability range of the nematic biaxial phase, the answer seems to be negative. In all cases we have found that the stabilisation of the smectic biaxial phases was greater than that of the biaxial nematic, with the effect of actually non-increasing or more commonly reducing the existence window of this much sought phase. We believe this finding, although not optimistic, can be a useful hint to synthetic chemists in choosing their molecular design strategy.

\section{Acknowledgements}

We thank EU-STREP “Biaxial Nematic Devices" (BIND) FP7216025, and MIUR-PRIN national project "Novel order systems for high response molecular devices" (2009N5JH4F) for financial support, and the Italian Supercomputing centre CINECA for computer time.

\section{References}

1 L. J. Yu and A. Saupe, Phys. Rev. Lett., 1980, 45, 1000-1003.

2 C. Tschierske and D. J. Photinos, J. Mater. Chem., 2010, 20, 4263-4294.

3 G. R. Luckhurst, Nature, 2004, 430, 413-414.

4 R. Berardi, L. Muccioli, S. Orlandi, M. Ricci and C. Zannoni, J. Phys.: Condens. Matter, 2008, 20, 464101.

5 M. Lehmann, Liq. Cryst., 2011, 38, 1389-1405.

6 R. Berardi, L. Muccioli and C. Zannoni, J. Chem. Phys., 2008, 128, 024905.

7 M. J. Freiser, Phys. Rev. Lett., 1970, 24, 1041-1043.

8 F. Biscarini, C. Chiccoli, P. Pasini, F. Semeria and C. Zannoni, Phys. Rev. Lett., 1995, 75, 1803-1806.

9 M. P. Allen, Liq. Cryst., 1990, 8, 499-511.

10 R. Berardi and C. Zannoni, J. Chem. Phys., 2000, 113, 5971-5979. 
11 G. R. Luckhurst, Thin Solid Films, 2001, 393, 40-52.

12 F. Hessel and H. Finkelmann, Polym. Bull., 1986, 15, 349-352.

13 K. Severing and K. Saalwatcher, Phys. Rev. Lett., 2004, 92, 125501.

14 K. Severing, E. Stibal-Fischer, A. Hasenhindl, H. Finkelmann and K. Sallwatcher, J. Phys. Chem. B, 2006, 110, 15680.

15 F. Brömmel, W. Stille, H. Finkelmann and A. Hoffmann, Soft Matter, 2011, 7, 2387-2401.

16 F. Brömmel, P. Benzie, G. J. Osterwinter, D. Kramer, H. Finkelmann, S. Elston and A. Hoffmann, Soft Matter, 2013, 9, 2646-2651.

17 E. van den Pol, A. V. Petukhov, D. Thies-Weesie, D. V. Byelov and G. J. Vroege, Phys. Rev. Lett., 2009, 103, 258301.

18 L. A. Madsen, T. J. Dingemans, M. Nakata and E. T. Samulski, Phys. Rev. Lett., 2004, 92, 145505.

19 B. R. Acharya, A. Primak and S. Kumar, Phys. Rev. Lett., 2004, 92, 145506.

20 K. Merkel, A. Kocot, J. K. Vij, R. Korlacki, G. H. Mehl and T. Meyer, Phys. Rev. Lett., 2004, 93, 237801.

21 N. Madhusudana, Mol. Cryst. Liq. Cryst., 2004, 409, 371-387.

22 R. Berardi, S. Orlandi and C. Zannoni, Chem. Phys. Lett., 1996, 261, 357.

23 B. Mettout, P. Tolédano, H. Takezoe and J. Watanabe, Phys. Rev. E: Stat., Nonlinear, Soft Matter Phys., 2002, 66, 031701.

24 R. Berardi, S. Orlandi and C. Zannoni, Phys. Rev. E: Stat., Nonlinear, Soft Matter Phys., 2003, 124, 041708.

25 K. Satoh, S. Mita and S. Kondo, Chem. Phys. Lett., 1996, 255, 99-104.

26 E. G. A. Bródka and K. Pasterny, Chem. Phys. Lett., 1997, 267, 557-562.

27 M. Houssa, L. F. Rull and S. C. McGrother, J. Chem. Phys., 1998, 109, 9529-9542.

28 J. S. van Duijneveldt, A. Gil-Villegas, G. Jackson and M. P. Allen, J. Chem. Phys., 2000, 112, 9092-9104.

29 R. Berardi, S. Orlandi and C. Zannoni, J. Chem. Soc., Faraday Trans., 1997, 93, 1493-1496.

30 R. Berardi, S. Orlandi and C. Zannoni, Liq. Cryst., 2005, 32, 1427-1436.

31 G. Tiberio, L. Muccioli, R. Berardi and C. Zannoni, ChemPhysChem, 2009, 10, 125-136.
32 A. Januszko, K. L. Glab, P. Kaszynski, K. Patel, R. A. Lewis, G. H. Mehl and M. D. Wand, J. Mater. Chem., 2006, 16, 3183-3192.

33 B. Ringstrand and P. Kaszynski, J. Mater. Chem., 2010, 20, 9613-9615.

34 D. Dunmur and K. Toriyama, in Physical Properties of Liquid Crystals, ed. D. Demus, J. W. Goodby, G. W. Gray, H.-W. Spiess and V. Vill, Wiley-VCH, Weinheim, 1999, pp. 129-150.

35 J. G. Gay and B. J. Berne, J. Chem. Phys., 1981, 74, 3316-3319.

36 R. Berardi, C. Fava and C. Zannoni, Chem. Phys. Lett., 1995, 236, 462-468.

37 R. Berardi, C. Fava and C. Zannoni, Chem. Phys. Lett., 1998, 297, 8-14.

38 M. E. Rose, Elementary Theory of Angular Momentum, Dover, New York, 1995.

39 C. Zannoni and M. Guerra, Mol. Phys., 1981, 44, 849-869.

40 I. G. Tironi, R. Sperb, P. E. Smith and W. F. van Gunsteren, J. Chem. Phys., 1995, 102, 5451-5459.

41 R. Berardi, J. Lintuvuori, M. Wilson and C. Zannoni, J. Chem. Phys., 2011, 135, 134119.

42 R. Berardi and C. Zannoni, Soft Matter, 2012, 8, 2017-2025.

43 H. J. C. Berendsen, J. P. M. Postma, W. F. van Gunsteren, A. Di Nola and J. R. Haak, J. Chem. Phys., 1984, 81, 3684-3690. 44 R. Berardi, S. Orlandi and C. Zannoni, Phys. Chem. Chem. Phys., 2000, 2, 2933-2942.

45 R. Berardi and C. Zannoni, Mol. Cryst. Liq. Cryst., 2003, 396(PN Part 1), 177-186.

46 M. Ricci, R. Berardi and C. Zannoni, Soft Matter, 2008, 4, 2030-2038.

47 F. Stillinger, Science, 1995, 267, 1935-1939.

48 A. G. Vanakaras and D. J. Photinos, J. Chem. Phys., 2008, 128, 154512.

49 O. Francescangeli, V. Stanic, S. I. Torgova, A. Strigazzi, N. Scaramuzza, C. Ferrero, I. P. Dolbnya, T. M. Weiss, R. Berardi, L. Muccioli, S. Orlandi and C. Zannoni, Adv. Funct. Mater., 2009, 19, 2592-2600.

50 M. P. Taylor and J. Herzfeld, Phys. Rev. A, 1991, 44, 3742-3751. 51 R. Berardi, M. Ricci and C. Zannoni, ChemPhysChem, 2001, 2, 443-447. 